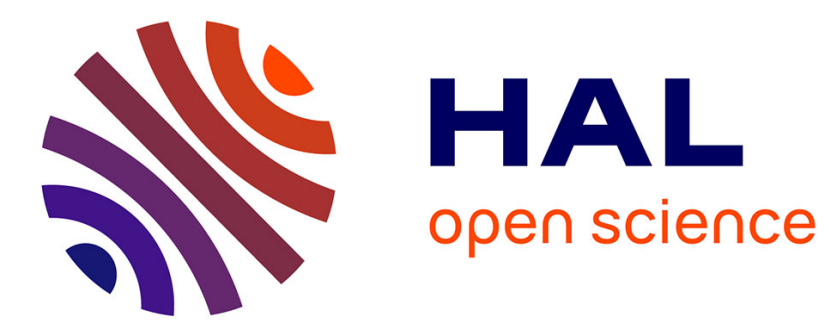

\title{
Fast Cramer-Shoup Cryptosystem
}

\author{
Pascal Lafourcade, Léo Robert, Demba Sow
}

\section{To cite this version:}

Pascal Lafourcade, Léo Robert, Demba Sow. Fast Cramer-Shoup Cryptosystem. 18th International Conference on Security and Cryptography, SECRYPT 2021, Jul 2021, Online, France. 10.5220/0010580607660771. hal-03419423

\section{HAL Id: hal-03419423 \\ https://hal.science/hal-03419423}

Submitted on 8 Nov 2021

HAL is a multi-disciplinary open access archive for the deposit and dissemination of scientific research documents, whether they are published or not. The documents may come from teaching and research institutions in France or abroad, or from public or private research centers.
L'archive ouverte pluridisciplinaire HAL, est destinée au dépôt et à la diffusion de documents scientifiques de niveau recherche, publiés ou non, émanant des établissements d'enseignement et de recherche français ou étrangers, des laboratoires publics ou privés. 


\title{
Fast Cramer-Shoup Cryptosystem
}

\author{
Pascal Lafourcade ${ }^{1} \mathbb{1}^{\mathrm{a}}$, Léo Robert ${ }^{1} \mathbb{1}^{\mathrm{b}}$ and Demba Sow ${ }^{2} \mathbb{C O}^{\mathrm{c}}$ \\ ${ }^{1}$ Université Clermont Auvergne, LIMOS CNRS (UMR 6158), Campus des Cézeaux, Aubière, France \\ ${ }^{2}$ LACGAA, Université Cheikh Anta Diop de Dakar, Sénégal \\ \{pascal.lafourcade,leo.robert $\} @$ uca.fr,dembal.sow@ucad.edu.sn
}

Keywords: Public Key Encryption, Cramer-Shoup, IND-CCA2.

\begin{abstract}
Cramer-Shoup was the first practical adaptive CCA-secure public key encryption scheme. We propose a faster version of this encryption scheme, called Fast Cramer-Shoup. We show empirically and theoretically that our scheme is faster than three versions proposed by Cramer-Shoup in 1998. We observe an average gain of $60 \%$ for the decryption algorithm. We prove the IND-CCA2 security of our scheme. The proof only relies on intractability assumptions like DDH.
\end{abstract}

\section{Introduction}

Provable security is an important issue in modern cryptography. It allows us to formally prove the security of the encryption schemes by reduction to difficult problems such as discrete logarithm problem (DL), Computational Decisional Diffie-Hellman problem $(\mathrm{CDH})$, Decision Diffie-Hellman problem (DDH) (Boneh, 1998; Joux and Guyen, 2006) or the quadratic residuosity problem. For instance, the DDH problem is used to prove the IND-CPA security of the ElGamal encryption scheme (Elgamal, 1985). In order to have security against adaptive chosen ciphertext attacks (IND-CCA2), a notion introduced in 1991 by Dolev et al. (Dolev et al., 1991), Cramer and Shoup proposed in 1998 an encryption scheme (Cramer and Shoup, 1998) that has a verification mechanism in the decryption algorithm to avoid malleability of the ciphertext and also uses one hash function.

Fujisaki and Okamoto in (Fujisaki and Okamoto, 1999) proposed a generic conversion from any INDCPA cryptosystem into an IND-CCA2 one, in the random oracle model (ROM) (Bellare and Rogaway, 1993). However the design of an IND-CCA2 encryption scheme is not easy, as the story of Optimal Asymmetric Encryption Padding (OAEP) (Bellare and Rogaway, 1994; Pointcheval, 2011) can show. After a first try by Bellare and Rogaway (Bellare and Rogaway, 1994 ) in 1995, V. Shoup found a problem in (Shoup,

\footnotetext{
a (iD https://orcid.org/0000-0002-4459-511X

b (iD) https://orcid.org/0000-0002-9638-3143

c (iD) https://orcid.org/0000-0002-1917-2051
}

2001), that was fixed in (Phan and Pointcheval, 2004; Pointcheval, 2011). Finally to conclude the story of OAEP, an computer verified proof has been made in (Barthe et al., 2011).

Our goal is to design a faster version of CramerShoup scheme. For this, we use the approach proposed in (Sow and Sow, 2011) to improve the decryption algorithm of ElGamal (Elgamal, 1985).

Contributions: Our main aim is to improve the efficiency of the Cramer-Shoup public key scheme:

1. We design a public key cryptosystem, called Fast Cramer-Shoup, based on the Generalized ElGamal encryption scheme (Sow and Sow, 2011).

2. We implemented all these schemes with GMP (Granlund, 2020) to demonstrate that Fast Cramer-Shoup is the fastest one with a gain of $60 \%$ for decryption algorithm .

3. We prove its security against the adaptive chosen ciphertext attack (IND-CCA2) under the (DDH) assumption.

Related works: Shoup and Gennaro (Shoup and Gennaro, 1998) give two ElGamal-like practical threshold cryptosystems that are secure against adaptive chosen ciphertext attack in the random oracle model. They use $H\left(h^{r}\right) \oplus m$ to encrypt the message $m$, unfortunately the trick of Sow et al. (Sow and Sow, 2011) cannot be applied in this case.

In (Cramer and Shoup, 2002), the authors proposed a construction by considering an algebraic primitive called universal hash proof systems. They 
showed that this framework yields not only the original DDH-based Cramer-Shoup's scheme but also encryption schemes based on quadratic residuosity and on Paillier's assumption (Paillier, 1999).

In 2011, a modified variant of ElGamal's encryption scheme was presented (Sow and Sow, 2011), and it is called Generalized ElGamal's encryption scheme. This version is faster than ElGamal, encryption algorithm is the same as ElGamal's encryption mechanism, the key generation algorithm is slower but the decryption process is faster. We adapt this idea to improve Cramer and Shoup's encryption scheme.

Outline: In Section 2, we propose our public key cryptosystem, called Fast Cramer-Shoup. In Section 3, we present the result of our empirically performance comparison and our complexity analysis for the key generation, encryption and decryption algorithms for all versions of Cramer-Shoup.

\section{Fast Cramer-Shoup's Encryption Scheme}

We present our Fast Cramer-Shoup's scheme and prove that it is IND-CCA2 secure. Let us recall some notions and definitions like the set of non-negative integers $\mathbf{Z}_{\geq 0}$, a security parameter $\lambda$, a group description $\Gamma$, a computational group scheme $\mathcal{G}$, a probability distribution of group descriptions $S_{\lambda}$, hash functions (HF), target collision resistant (TCR) assumption for hash function (HF), some random variables as Coins used in the following are defined in (Cramer and Shoup, 2003) (see also (Naor and Yung, 1989)).

\section{Fast Key Generation Algorithm:}

G1 : On input $1^{\lambda}$ for $\lambda \in \mathbf{Z}_{\geq 0}$, select a group $\hat{G}$, along with a prime-order subgroup $G$ and choose a generator $g_{2} \in G$ of order $q$.

G2: Pick random elements $x, y, k, t \in \mathbb{Z}_{q}$ with $\log _{2}(t)=\frac{\log _{2}(q)}{2}$, and compute $w^{\prime}, z \in \mathbb{Z}_{q}$ such that $k q=t w^{\prime}+z$ and then compute $w \equiv w^{\prime}(\bmod q)$.

G3 : Compute $g_{1}=g_{2}^{w}, c=g_{2}^{w x}, d=g_{2}^{w y}$ and $h=g_{2}^{z}$.

G4 : Choose a hash function $H \stackrel{R}{\leftarrow} \mathbf{H F}$.

G5 : Return $(p k, s k)$, where $p k=\left(\Gamma, H, g_{1}, c, d, h\right)$ and $s k=(\Gamma, H, t, x, y, z)$.

\section{Fast Encryption Algorithm:}

E1 : Choose a random element $r \in \mathbb{Z}_{q}$ and compute,

E2 : $u_{1}=g_{1}^{r} ; \mathbf{E 3}: u_{2}=g_{2}^{r} ; \mathbf{E 4}: u_{3}=h^{r} ; \mathbf{E 5}: e=u_{3} m$;

E6 : $\alpha=H\left(u_{1}, u_{2}, e\right)$;

E7 : $v=c^{r} d^{r \alpha}$ and output $\psi=\left(u_{1}, u_{2}, e, v\right)$.
Fast Decryption Algorithm:

D1 : Parse $\psi \leftarrow\left(u_{1}, u_{2}, e, v\right) \in G^{4}$; output reject if $\psi$ is not of this form.

D2' ${ }^{\prime}$ : Test if $u_{1}$ and $u_{2}$ belong to $G$; reject otherwise.

D3 : Compute $\alpha=H\left(u_{1}, u_{2}, e\right)$.

D4' : Test if $u_{1}^{t} u_{2}^{z}=1$ and $v=u_{1}^{x+y \alpha}$; otherwise reject.

$\mathbf{D 5}^{\prime}$ : Compute $\beta=u_{1}^{t}$; D6 : Output $m=\beta e$.

Correctness Verification: We have $\beta u_{2}^{z}=u_{1}^{t} u_{2}^{z}=$ $\left(g_{1}^{r}\right)^{t} g_{2}^{r z}=\left(g_{2}^{w r}\right)^{t} g_{2}^{r z}=g_{2}^{r(t w+z)}=g_{2}^{r k q}=1$ since the order of $g_{2}$ is $q$ and $u_{1}^{x+y \alpha}=\left(g_{1}^{r}\right)^{x+y \alpha}=\left(g_{2}^{w r}\right)^{x+y \alpha}=$ $\left(g_{2}^{w x}\right)^{r}\left(g_{2}^{w y}\right)^{r \alpha}=c^{r} d^{r \alpha}=v$.

Decryption: The decryption message is $\beta e=$ $u_{1}^{t} e=g_{2}^{w r t} g_{2}^{z r} m=g_{2}^{r(t w+z)} m=g_{2}^{r k q} m=m$, since the order of $g_{2}$ is $q$.

\subsection{Security Proof of Fast Cramer-Shoup Scheme}

As CS1's proof in (Cramer and Shoup, 2003), to prove that Fast Cramer-Shoup (FCS) is secure against adaptive chosen ciphertext attack if the DDH assumption holds for $\mathcal{G}$ and the TCR assumption holds for HF, we need some notions.

Suppose PKE is a public-key encryption scheme that uses a group scheme in the following natural way: on input $1^{\lambda}$, the key generation algorithm runs the sampling algorithm of the group scheme on input $1^{\lambda}$, yielding a group description $\Gamma$. For a given probabilistic, polynomial-time oracle query machine $\mathcal{A}, \lambda \in \mathbf{Z}_{>0}$, and group description $\Gamma$, let us define $\operatorname{AdvCCA} \operatorname{PKE}, \mathcal{A}(\lambda \mid \Gamma)$ to be $\mathcal{A}$ 's advantage in an adaptive chosen ciphertext attack where the key generation algorithm uses the given value of $\Gamma$, instead of running the sampling algorithm of the group scheme. For all probabilistic, polynomial-time oracle query machines $\mathcal{A}$, for all $\lambda \in \mathbf{Z}_{\geq 0}$, let $Q_{\mathcal{A}}(\lambda)$ be an upper bound on the number of decryption oracle queries made by $\mathcal{A}$ on input $1^{\lambda}$. We assume that $Q_{\mathcal{A}}(\lambda)$ is a strict bound in the sense that it holds regardless of the probabilistic choices of $\mathcal{A}$, and regardless of the responses to its oracle queries from its environment.

Theorem 2.1. The Fast Cramer-Shoup is secure against adaptive chosen ciphertext attack if:

1. the DDH assumption holds for $\mathcal{G}$;

2. and the target collision resistance (TCR) assumption holds for $\mathbf{H F}$.

In particular, for all probabilistic, polynomial-time oracle query machines $\mathcal{A}$, for all $\lambda \in \mathbf{Z}_{\geq 0}$, and all $\Gamma\left[\hat{G}, G, g_{2}, q\right] \in\left[\mathbf{S}_{\lambda}\right]$, we have

$\left|\operatorname{AdvCCA}_{\mathrm{FCS}, \mathcal{A}}(\lambda \mid \Gamma)-\operatorname{AdvCCA}_{\mathrm{CS} 1, \mathcal{A}}(\lambda \mid \Gamma)\right| \leq Q_{\mathcal{A}}(\lambda) / q$. 
Description of games: Suppose that $p k=$ $\left(\Gamma, H, g_{1}, c, d, h\right)$ and $s k=(\Gamma, H, t, x, y, z)$. Let $w=\log _{g_{2}} g_{1}$, and define $x, y, z \in \mathbb{Z}_{q}$ as follows: $x=x_{1}+x_{2} w, y=y_{1}+y_{2} w$ and $z=z_{1}+z_{2} w$. We have $x=\log _{g_{2}} c, y=\log _{g_{2}^{w}} d$, and $z=\log _{g_{2}} h$.

As a notation convention, whenever a particular ciphertext is under consideration in some context, the following values are also implicitly defined in that context: $u_{1}, u_{2}, u_{3}, e, v \in G$ where $\psi=\left(u_{1}, u_{2}, e, v\right)$ and $u_{3}=u_{2}^{z}$; the random $r \in \mathbb{Z}_{q}$, where $r=\log _{g_{1}^{w}} u_{1}$.

For the target ciphertext $\psi^{*}$, we also denote by $u_{1}^{*}, u_{2}^{*}, u_{3}^{*}, e^{*}, v^{*} \in G$ and $r^{*} \in \mathbb{Z}_{q}$ the corresponding values. The probability space defining the attack game is then determined by the following, mutually independent, random variables: the coin tosses Coins of $\mathcal{A}$; the values $H, w, x_{1}, x_{2}, y_{1}, y_{2}, z_{1}, z_{2}$ generated by the key generation algorithm; the values $\sigma \in\{0,1\}$ and $r^{*} \in \mathbb{Z}_{q}$ generated by the encryption oracle.

$\mathbf{G}_{0}$ : Original attack game, let $\hat{\sigma} \in\{0,1\}$ be the output of $\mathcal{A}$ and $T_{0}$ the event $\sigma=\hat{\sigma}$, so $\operatorname{AdvCCA}_{\mathbf{F C S}, \mathcal{A}}(\lambda \mid \Gamma)=\left|\operatorname{Pr}\left[T_{0}\right]-1 / 2\right|$

$\mathbf{G}_{1}$ : We now modify game $\mathbf{G}_{0}$ to obtain game $\mathbf{G}_{1}$. These two games are identical, except that instead of using the encryption algorithm as given to compute the target ciphertext $\psi^{*}$, we use a modified encryption algorithm, in which steps E4 and E7 are replaced by $\mathbf{E 4 ^ { \prime }}: u_{3}=u_{2}^{z}$ and $\mathbf{E} 7^{\prime}: v=u_{1}^{x+y \alpha}$. The change we have made is purely conceptual. The values of $u_{3}^{*}$ and $v^{*}$ are exactly the same in game $\mathbf{G}_{1}$ as they were in $\mathbf{G}_{0}$ so $\operatorname{Pr}\left[T_{1}\right]=\operatorname{Pr}\left[T_{0}\right]$

$\mathbf{G}_{2}$ : We modify the encryption oracle, replacing step $\mathbf{E 3}$ by $\mathbf{E 3}^{\prime}: \hat{r} \stackrel{R}{\leftarrow} \mathbb{Z}_{q} \backslash\{r\} ; u_{2} \leftarrow g_{2}^{\hat{r}}$

Lemma 2.2. There exists a probabilistic algorithm $\mathcal{A}_{1}$, whose running time is essentially the same as that of $\mathcal{A}$, such that

$$
\left|\operatorname{Pr}\left[T_{2}\right]-\operatorname{Pr}\left[T_{1}\right]\right| \leq \operatorname{AdvDDH}_{\mathcal{G}, \mathcal{A}_{1}}(\lambda \mid \Gamma)+3 / q .
$$

$\mathbf{G}_{3}$ : We modify the decryption algorithm, replacing steps D4 and D5 with D4' : Test if $u_{1}=u_{2}^{w}$ and $v=u_{1}^{x+y \alpha}$; output reject and halt if this is not the case. $\mathbf{D 5}^{\prime}: u_{3}=u_{2}^{z}$. Note that the decryption oracle now make use of $w$, but does not make use of $x_{1}, x_{2}, y_{1}, y_{2}, z_{1}, z_{2}$, except indirectly through the values $x, y, z$. Now, let $R_{3}$ be the event that in game $\mathbf{G}_{3}$, some ciphertext $\psi$ is submitted to the decryption oracle that is rejected in step $\mathbf{D 4}^{\prime}$ but that would have passed the test in step D4. Note that if a ciphertext passes the test in $\mathbf{D 4 ^ { \prime }}$, it would also have passed the test in D4. It is clear that games $\mathbf{G}_{2}$ and $\mathbf{G}_{3}$ proceed identically until the event $R_{3}$ occurs. In particular, the events $T_{2} \wedge \neg R_{3}$ and $T_{3} \wedge \neg R_{3}$ are identical. So by difference lemma $\left|\operatorname{Pr}\left[T_{3}\right]-\operatorname{Pr}\left[T_{2}\right]\right| \leq \operatorname{Pr}\left[R_{3}\right]$, and so it suffices to bound $\operatorname{Pr}\left[R_{3}\right]$. We introduce auxiliary games $\mathbf{G}_{4}$ and $\mathbf{G}_{5}$ below to do this.
$\mathbf{G}_{4}:$ We replace step $\mathbf{E 5}$ by $\mathbf{E 5}^{\prime}: r \stackrel{R}{\leftarrow} \mathbb{Z}_{q} ; e \leftarrow g^{\gamma}$ so $\operatorname{Pr}\left[T_{4}\right]=1 / 2$, since in game $\mathbf{G}_{4}$, the variable $\sigma$ is never used. Define the event $R_{4}$ to be the event in game $\mathbf{G}_{4}$ analogous to the event $R_{3}$ in game $\mathbf{G}_{3}$; that is, $R_{4}$ is the event that in game $\mathbf{G}_{4}$, some ciphertext $\psi$ is submitted to the decryption oracle that is rejected in step $\mathbf{D} \mathbf{4}^{\prime}$ but that would have passed the test in step D4. We show that this modification has no effect; more precisely: $\operatorname{Pr}\left[T_{4}\right]=\operatorname{Pr}\left[T_{3}\right]$, and $\operatorname{pr}\left[r_{4}\right]=\operatorname{pr}\left[r_{3}\right]$

$\mathbf{G}_{5}$. We modify the decryption oracle with a special rejection rule: if the adversary submits a ciphertext $\psi$ for decryption at a point in time after the encryption oracle has been invoked, such that $\left(u_{1}, u_{2}, e\right) \neq\left(u_{1}^{*}, u_{2}^{*}, e^{*}\right)$ but $\alpha=\alpha^{*}$, then the decryption oracle immediately outputs reject and halts (before executing step $\mathbf{D 4 ^ { \prime }}$ ). to analyze this game, we define two events. first, we define the event $C_{5}$ to be the event that the decryption oracle in game $\mathbf{G}_{5}$ rejects a ciphertext using the special rejection rule. We define the event $R_{5}$ to be the event in game $\mathbf{G}_{5}$ that some ciphertext $\psi$ is submitted to the decryption oracle that is rejected in step $\mathbf{D 4 ^ { \prime }}$ but that would have passed the test in step D4. note that such a ciphertext is not rejected by the special rejection rule, since that rule is applied before step $\mathbf{D 4}^{\prime}$ is executed. Now, it is clear that games $\mathbf{G}_{4}$ and $\mathbf{G}_{5}$ proceed identically until event $C_{5}$ occurs. By difference lemma, we have $\left|\operatorname{Pr}\left[R_{5}\right]-\operatorname{Pr}\left[R_{4}\right]\right| \leq \operatorname{Pr}\left[C_{5}\right]$. Now, if event $C_{5}$ occurs with non-negligible probability, we immediately get an algorithm that contradicts the target collision resistance assumption;

Lemma 2.3. There exists a probabilistic algorithm $\mathcal{A}_{2}$, whose running time is essentially the same as that of $\mathcal{A}$, such that:

$$
\operatorname{Pr}\left[C_{5}\right] \leq \operatorname{AdvTCR}_{\mathbf{H F}, \mathcal{A}_{2}}(\lambda \mid \Gamma)+1 / q .
$$

Finally, we show that $R_{5}$ occurs with negligible probability, based on information theoretic considerations: we have $\operatorname{Pr}\left[R_{5}\right] \leq Q_{\mathcal{A}}(\lambda) / q$.

Theorem 2.1. To prove this theorem, let us fix $\mathcal{A}, \lambda$, and $\Gamma\left[\hat{G}, G, g_{2}, q\right]$. Consider the attack game $\mathbf{G}_{0}$ as defined above (or see $\$ 6.2$ in (Cramer and Shoup, 2003)). This is the game that attacker $\mathcal{A}$ plays against the scheme FCS for the given values of $\lambda$ and $\Gamma$. We adopt all the notations conventions established at the beginning of $\S 6.2$ in (Cramer and Shoup, 2003). We now modify game $\mathbf{G}_{0}$ to obtain a new game $\mathbf{G}_{\mathbf{F C S}}$.

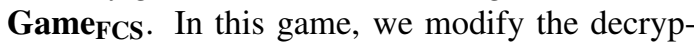
tion oracle so that in place of steps D4 and D5 in CS1, we execute steps $\mathbf{D 4}^{\prime}$ and $\mathbf{D 5}^{\prime}$ as in the scheme FCS. (Note that in the FCS encryption algorithm the random parameter generated is $r$ instead of $u$ in CS1 scheme. So the parameter $u^{*}$ in CS1 corresponds 
to $r^{*}$ here). We emphasize that in game $\mathbf{G}_{\mathbf{F C S}}$, we have $x=x_{1}+x_{2} w, y=y_{1}+y_{2} w$, and $z=z_{1}+z_{2} w$, where $w, x_{1}, x_{2}, y_{1}, y_{2}, z_{1}$, and $z_{2}$ are generated by the key generation algorithm of CS1.

Let $T_{\mathbf{F C S}}$ be the event that $\sigma=\sigma^{\prime}$ in game $\mathbf{G}_{\mathbf{F C S}}$. We remind the reader that games $\mathbf{G}_{0}$ and $\mathbf{G}_{\mathbf{F C S}}$ all operate on the same underlying probability space: all of the variables Coins, $H, w, x_{1}, x_{2}, y_{1}, y_{2}, z_{1}, z_{2}, \sigma, r^{*}$ that ultimately determine the events $T_{0}$, and $T_{\mathbf{F C S}}$ have the same values in games $\mathbf{G}_{0}$ and $\mathbf{G}_{\mathbf{F C S}}$; all that changes is the functional behavior of the decryption oracle. It is straightforward to verify that $\operatorname{AdvCCA}_{\mathbf{F C S}, \mathcal{A}}(\lambda \mid \Gamma)=\left|\operatorname{Pr}\left[T_{\mathbf{F C S}}\right]-1 / 2\right|$

Let us define the event $R_{\text {FCS }}$ to be the event that some ciphertext is rejected in game $\mathbf{G}_{\mathbf{F C S}}$ in step $\mathbf{D} \mathbf{4}^{\prime}$ that would have passed the test in D4 in CS1 scheme in (Cramer and Shoup, 2003). It is clear that games $\mathbf{G}_{0}$ and $\mathbf{G}_{\mathbf{F C S}}$ all proceed identically until event $R_{\mathbf{F C S}}$ occurs. In particular, we have the events $T_{0} \wedge \neg R_{\mathbf{F C S}}$, and $T_{\mathbf{F C S}} \wedge \neg R_{\mathbf{F C S}}$ are identical. So by difference lemma, we have $\left|\operatorname{Pr}\left[T_{0}\right]-\operatorname{Pr}\left[T_{\mathbf{F C S}}\right]\right| \leq \operatorname{Pr}\left[R_{\mathbf{F C S}}\right]$. So it suffices to show that $\operatorname{Pr}\left[R_{\mathbf{F C S}}\right] \leq Q_{\mathcal{A}}(\lambda) / q$. To do this, for $1 \leq i \leq Q_{\mathcal{A}}(\lambda)$, let $R_{\mathrm{FCS}}^{(i)}$ be the event that there is an $i^{t h}$ ciphertext submitted to the decryption oracle in game $\mathbf{G}_{\mathbf{F C S}}$, and that this ciphertext is rejected in step D4', but would have passed the test in step D4 in CS1 scheme in (Cramer and Shoup, 2003). The bound will follow immediately from the following lemma.

Lemma 2.4. For all $1 \leq i \leq Q_{\mathcal{A}}(\lambda)$, we have $\operatorname{Pr}\left[R_{\mathbf{F C S}}^{(i)}\right] \leq 1 / q$.

Proof. The proof of this lemma is almost identical to Lemma 10 in (Cramer and Shoup, 2003). Note that in game $\mathbf{G}_{\mathbf{F C S}}$, the encryption oracle uses the "real" encryption algorithm, and so itself does not leak any additional information about $\left(x_{1}, x_{2}, y_{1}, y_{2}\right)$. This is in contrast to game $\mathbf{G}_{5}$, where the encryption oracle does leak additional information.

Fix $1 \leq i \leq Q_{\mathcal{A}}(\lambda)$. Consider the quantities: $X:=$ (Coins, $\left.H, w, z, \sigma, r^{*}\right)$ and $X^{\prime}:=(x, y)$. The values of $X$ and $X^{\prime}$ completely determine the adversary's entire behavior in game $\mathbf{G}_{5}$, and hence determine if there is an $i^{\text {th }}$ decryption oracle query, and if so, the value of the corresponding ciphertext. Let us call $X$ and $X^{\prime}$ relevant if for these values of $X$ and $X^{\prime}$, there is an $i^{\text {th }}$ decryption oracle query, and the corresponding ciphertext passes steps D1 and D2 in CS1 scheme in (Cramer and Shoup, 2003).

It will suffice to prove that conditioned on any fixed, relevant values of $X$ and $X^{\prime}$, the probability that $R_{\text {FCS }}^{(i)}$ occurs is bounded by $1 / q$. The remainder of the argument is exactly as in Lemma 10 in (Cramer and

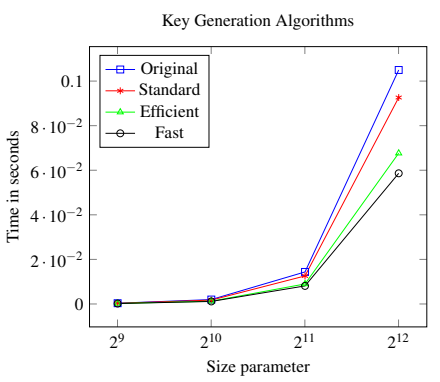

Figure 1: Average execution time for key generation algorithms depending of the security parameter size.

\begin{tabular}{l|c|c|c|c|}
\hline \multicolumn{5}{c}{ Key Generation } \\
\hline Number of & Original & Standard & Efficient & Fast \\
\hline Generator & 2 & 2 & $\mathbf{1}$ & $\mathbf{1}$ \\
\hline Random & 6 & 5 & $\mathbf{4}$ & $\mathbf{4}$ \\
\hline Multiplication & 3 & 2 & $\mathbf{0}$ & 3 \\
\hline Exponentiation & 6 & 5 & $\mathbf{4}$ & $\mathbf{4}$ \\
\hline
\end{tabular}

Table 1: Comparison of Original, Standard, Efficient and Fast Cramer-Shoup (minimum in bold).

Shoup, 2003), except using $X, X^{\prime}$, and the notion of relevant as defined here.

\section{Performances Evaluation}

We study the complexity and the performance of Fast Cramer-Shoup encryption scheme with the following variants of Cramer-Shoup encryption scheme: Original CS, Standard CS, Efficient CS, Fast CramerShoup protocols. We study the number of operations performed for key generation, encryption and decryption. We present complexity results in Table 1 to Table 3 . We also implemented those with the library GMP (Granlund, 2020).

The tests are performed with security parameters of size 512, 1024, 2048 and 4096 bits. The average execution time of all schemes is carried out under the same conditions in terms of generation of the values and sizes of the security parameters. Indeed, given the size of a security parameter, we perform 1000 trials with new parameters: a prime number and some random plaintexts that are generated for each trial. Then, we measure the execution time during one execution of each algorithm. Finally, we compute the mean value for each algorithm (we do not present the corresponding standard deviations since they are very low).

Key Generation Algorithms. By studying the complexity of the key generation algorithm of Table 1, we notice that Fast $C S$ and Efficient $C S$ are the 


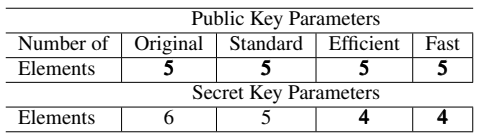

Table 2: Comparison for key parameters.

fastest in terms of number of operations. The difference lies in the multiplication where Fast $C S$ has three of them while Efficient $C S$ has none. One would expect a longer execution time for Fast CS but this is not the case (Figure 1). It can be explained by the fact that the Fast $C S$ key generation algorithm computes an Euclidean division leading to $k q=w t+z$ where $k$ is generated in $\mathbb{Z}_{q}$. Hence its size is the same as $q$ but $t$ is generated in $\mathbb{Z}_{\sqrt{q}}^{*}$ and its size is half of $q$. Since $z$ is the remainder of the euclidean division, its order size is the same as $t$. Thus the cost for computing $h=g^{z}$ is half of the cost for computing $h=g^{z}$ where $z$ has an order size equal to the size of $q$. This gain seems to be compensated with the three additional multiplications. To conclude for key generation, the two fastest algorithms are Efficient $C S$ and Fast CS, while the two slowest are Original CS and Standard CS. Moreover, the number of secret parameters are also in favour of Efficient CS and Fast CS as depicted in Table 2.

Encryption Algorithms. We can see that all schemes have the same number of exponents, multiplication and generation of random number in the encryption algorithm. Thus the average execution time is the same. We observe empirically that timings are equal for all protocols since the number of computations are equals. In all protocols, the ciphertexts are mainly computed with parameters of the same order size. We do not give the curve results since the average execution time is the same in all cases.

Decryption Algorithms. We observe in Figure 2a that the fastest is Fast Cramer-Shoup followed by Efficient $C S$ which is not obvious when we are looking at the number of total computations.

Indeed there are two components in this algorithm, the verification phase and the actual decryption. The verification is used for checking the integrity of messages if the outputs are valid and the decryption computation is executed leading to retrieve the plaintext. In Figure 2a, we observe that Fast Cramer-Shoup has a faster execution time. For verification phase, we observe in Figure $2 b$ that the average execution time of the Fast CS and Efficient CS protocols have the same average execution time. In both cases the second verification is the same. The difference is then on the first verification. The Efficient $C S$ protocol uses one modular exponent, $u_{1}^{w}=u_{2}$; while

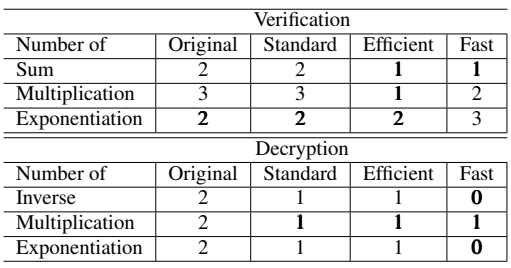

Table 3: Comparison for decryption algorithms.

ours uses two modular exponents, $\beta u_{2}^{z}=u_{1}^{w} u_{2}^{z}=1$. Despite the result given in Table 3 , there is actually no difference between those two computations in terms of execution time since elements $t$ and $z$ of Fast $C S$ have their size equal to half the size of $w$ in the $E f$ ficient CS. Hence, the overall execution time for the verification of our protocol Fast CS is the same as Efficient CS protocol. For the decryption phase, it is clear that Fast CS protocol is faster than Original for decryption since the number of exponents is reduced. We can also say from Table 3 that Fast CS is faster than Standard CS and Efficient $C S$ at decryption. We observe in Figure 2c that Standard CS and Efficient $C S$ have the same execution time. This result is expected since they both use the same computation with the same elements size, i.e., $e u_{1}^{-z}=m$. The latter computation uses one exponentiation and one inversion to end with a multiplication. The Fast CramerShoup decryption algorithm requires to recover $m$ as follows $\beta e=m$ where $\beta$ has been computed in the verification phase. Thus, the decryption consists of only one multiplication. The gain comes from the fact that we have one inverse less to compute, and also one exponentiation less to compute. The gain of the overall execution time of decryption phase for our protocol is more than $60 \%$. Note that the curve related to Fast Cramer-Shoup is not zero but since this curve is composed of only one multiplication, the comparison with a modular exponent, an inversion and a multiplication, is in favor of the stand alone multiplication.

\section{Conclusion}

We propose an IND-CCA2 Public Key cryptosystem called Fast Cramer-Shoup. It is an improvement of Cramer-Shoup scheme. We prove the IND-CCA2 security of this new scheme. We also implement in GMP (Granlund, 2020) our scheme to compare it to Cramer-Shoup schemes. In the future, we aim at applying our technique to other schemes that are based on Cramer-Shoup like for instance (Kurosawa and Trieu Phong, 2014; Abdalla et al., 2015). 


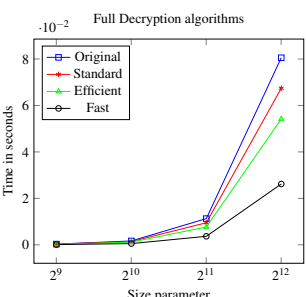

(a) Full decryption.

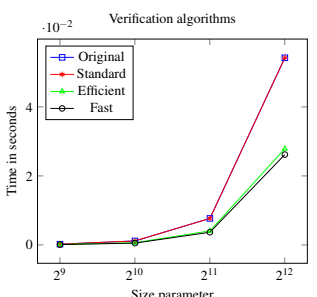

(b) Verification phases.

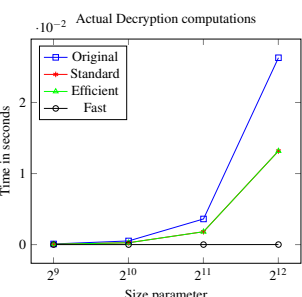

(c) Decryption phases.

Figure 2: Average execution time for decryption algorithms.

\section{REFERENCES}

Abdalla, M., Benhamouda, F., and Pointcheval, D. (2015). Public-key encryption indistinguishable under plaintext-checkable attacks. In Katz, J., editor, Public-Key Cryptography - PKC 2015, pages 332352, Berlin, Heidelberg. Springer Berlin Heidelberg.

Barthe, G., Grégoire, B., Lakhnech, Y., and Zanella Béguelin, S. (2011). Beyond provable security. Verifiable IND-CCA security of OAEP. In Topics in Cryptology - CT-RSA 2011, volume 6558 of Lecture Notes in Computer Science, pages 180-196. Springer.

Bellare, M. and Rogaway, P. (1993). Random oracles are practical: A paradigm for designing efficient protocols. In Proceedings of the 1st ACM Conference on Computer and Communications Security, CCS 93, page 6273, New York, NY, USA. Association for Computing Machinery.

Bellare, M. and Rogaway, P. (1994). Optimal asymmetric encryption. In Santis, A. D., editor, Advances in Cryptology - EUROCRYPT '94, Workshop on the Theory and Application of Cryptographic Techniques, Perugia, Italy, May 9-12, 1994, Proceedings, volume 950 of Lecture Notes in Computer Science, pages 92-111. Springer.

Boneh, D. (1998). The decision diffie-hellman problem. In In Proceedings of the Third Algorithmic Number Theory Symposium, volume 1423, pages 48-63.

Cramer, R. and Shoup, V. (1998). A practical public key cryptosystem provably secure against adaptive chosen ciphertext attack. In Proc. of the 18th Annual International Cryptology Conference on Advances in Cryptology, crypto'98, pages 13-25.

Cramer, R. and Shoup, V. (2003). Design and analysis of practical public-key encryption schemes secure against adaptive chosen ciphertext attack. SIAM Journal on Computing, 33(1):167-226.

Cramer, R. and Shoup, V. (May 2002). Universal hash proofs and a paradigm for adaptive chosen ciphertext secure public-key encryption. In In L. Knudsen, editor, Proceedings of Eurocrypt 2002, volume 2332 of LNCS, pages 45-64.

Dolev, D., Dwork, C., and Naor, M. (1991). Non-malleable cryptography. In Proceedings of the Twenty-Third Annual ACM Symposium on Theory of Computing, STOC 91, page 542552, New York, NY, USA. Association for Computing Machinery.
Elgamal, T. (1985). A public key cryptosystem and a signature scheme based on discrete logarithms. In CRYPTO, IT-31(4), volume 4, pages 469-472.

Fujisaki, E. and Okamoto, T. (1999). How to enhance the security of public-key encryption at minimum cost. In Public Key Cryptography, pages 53-68, Berlin, Heidelberg. Springer Berlin Heidelberg.

Granlund, T. (2020). GNU MP: The GNU Multiple Precision Arithmetic Library, 6.2.0 edition.

Joux, A. and Guyen, K. (2006). Separating decision diffiehellman to diffie-hellman in cryptographic groups.

Kurosawa, K. and Trieu Phong, L. (2014). Kurosawadesmedt key encapsulation mechanism, revisited. In Pointcheval, D. and Vergnaud, D., editors, Progress in Cryptology - AFRICACRYPT 2014, pages 51-68, Cham. Springer International Publishing.

Naor, M. and Yung, M. (1989). Universal one-way hash functions and their cryptographic applications. In In 21 st Annual ACM Symposium on Theory of Computing.

Paillier, P. (May 1999). Public-key cryptosystems based on composite degree residuosity classes. In In J. Stern, editor, Proceedings of Eurocrypt 1999, volume 1592 of LNCS, pages 223-38.

Phan, D. H. and Pointcheval, D. (2004). Oaep 3-round: A generic and secure asymmetric encryption padding. In Advances in Cryptology - ASIACRYPT 2004, 10th International Conference on the Theory and Application of Cryptology and Information Security, Jeju Island, Korea, December 5-9, 2004, Proceedings, volume 3329 of Lecture Notes in Computer Science, pages 63-77. Springer.

Pointcheval, D. (2011). OAEP: Optimal Asymmetric Encryption Padding, pages 882-884. Springer US, Boston, MA.

Shoup, V. (2001). Oaep reconsidered. In Proceedings of the 21st Annual International Cryptology Conference on Advances in Cryptology, CRYPTO 01, page 239259. Berlin, Heidelberg. Springer-Verlag.

Shoup, V. and Gennaro, R. (1998). Securing threshold cryptosystems against chosen ciphertext attack. In In Advances in Cryptology-Eurocrypt '98.

Sow, D. and Sow, D. (2011). A new variant of el gamal's encryption and signatures schemes. JP Journal of Algebra, Number Theory and Applications, 20(1):21-39. 